\title{
Qualimetrical Models of Integrated Assessment of Safety of Functioning of Military and Technical Systems
}

Vasiliy M. Umyvakin, Alexey A. Borodin and Alexey V. Shvets* Military Education and Research Centre of Military-Air Forces «Military-Air Academy Named After Professor N.E. Zhukovsky and Yu.A. Gagarin» 54a Starykh Bolshevikov Str., Voronezh, 394064, Russia

Received 12.01.2017, received in revised form 07.02.2017, accepted 01.03.2017

In work methodological issues of creation of qualimetrical models of integrated assessment of safety of functioning of military and technical systems within axiomatic (standard) approach to multicriteria tasks of acceptance of management decisions are considered.

Keywords: military and technical system, private and integrated qualimetrical estimates of functioning, assessment of safety of functioning.

Citation: Umyvakin V.M., Borodin A.A., Shvets A.V. Qualimetrical models of integrated assessment of safety of functioning of military and technical systems, J. Sib. Fed. Univ. Eng. technol., 2017, 10(2), 235-248. DOI: 10.17516/1999-494X-2017-10-2235-248.

(C) Siberian Federal University. All rights reserved

* Corresponding author E-mail address: umyvakin@mail.ru, lesha.borodin.76@mail.ru 


\title{
Квалиметрическая модель \\ интегральной оценки безопасности \\ функционирования военно-технических систем
}

\author{
В.М. Умывакин, \\ А.А. Бородин, А.В. Швец \\ Военный учебно-научный цеентр Военно-воздушных сил \\ «Военно-воздушная академия \\ имени профессора Н.Е. Жуковского и Ю.А. Гагарина» \\ Россия, 394064, Воронеж, ул. Старых Большевиков, 54 а
}

В статье рассматриваются методологические вопросы построения квалиметрических моделей интегральной оченки безопасности функционирования военно-технических систем в рамках аксиоматического (нормативного) подхода кмногокритериальныл задачам принятия управленческих решений.

Ключевые слова: военно-техническая система, частная и интегральная, квалиметрические оценки функционирования, оценки безопасности функционирования.

Для обеспечения безопасного функционирования военно-технических систем (ВТС) в условиях многокритериальности необходима интегральная оценка качества управленческих решений. Понятие «безопасность» не существует без антонима «опасность». Опасность - свойство, присущее любой сложной технической системе, реализующееся в виде прямого или косвенного ущерба для объекта воздействия. В дальнейшем под безопасностью функционирования ВТС понимается допустимый (приемлемый) уровень опасности (риска) - возможность (вероятность) недостижения требуемого качества управленческих решений. При этом интегральная оценка безопасности/опасности функционирования ВТС должна представлять собой некоторую «сводную» формулу, в которой «объединены» локальные относительные оценки по частным показателям качества управленческих решений; методики построения частных оценок должны входить составной частью в методику интегральной оценки; частные оценки и интегральная оценка должны допускать вероятностную интерпретацию; интегральная оценка должна быть адаптивной, позволяющей при необходимости расширять перечень частных показателей качества.

Приведем теоретическое обоснование необходимых свойств частных относительных оценок и интегральной оценки качества/некачественности (квалиметрических оценок) функционирования ВТС. Рассмотрим ситуацию, когда качество функционирования ВТС характеризуется только двумя частными показателями качества (ПК) - $\mathrm{y}_{1}$ и $\mathrm{y}_{2}$. Пусть $\mathrm{d}_{1}$ и $\mathrm{d}_{2}-$ соответствующие частные относительные квалиметрические оценки по этим ПК, a $d=d\left(d_{1}, d_{2}\right)$ - интегральная квалиметрическая оценка, которая рассматривается как результат некоторой операции над частными квалиметрическими оценками функционирования ВТС. Сформулируем основные априорные требования (аксиомы), лежащие в основе квалиметрического подхода к построению нелинейной (неаддитивной) интегральной квалиметрической оценки (табл. 1).

В табл. 2 указаны классы ассоциативных интегральных квалиметрических оценок в зависимости от предъявляемых аксиом. 
Таблица 1. Требования к интегральной квалиметрической оценке

\begin{tabular}{|c|c|c|c|}
\hline $\begin{array}{l}\text { № } \\
\Pi / \Pi\end{array}$ & $\begin{array}{l}\text { Требование к } \\
\text { интегральной } \\
\text { оценке }\end{array}$ & Формула & Комментарий \\
\hline 1 & $\begin{array}{l}\text { Коммутативность } \\
\text { (равноценность) }\end{array}$ & $\mathrm{d}\left(\mathrm{d}_{1}, \mathrm{~d}_{2}\right)=\mathrm{d}\left(\mathrm{d}_{2}, \mathrm{~d}_{1}\right)$ & $\begin{array}{l}\text { Одинаковая важность частных оценок } \mathrm{d}_{1} \text { и } \\
\mathrm{d}_{2} \text {, т.е. интегральная оценка не зависит от } \\
\text { порядка частных оценок, а зависит от всего } \\
\text { их множества }\end{array}$ \\
\hline 2 & $\begin{array}{l}\text { Ассоциативность } \\
\text { (иерархическая } \\
\text { одноуровненность) }\end{array}$ & $\begin{array}{l}\mathrm{d}\left(\mathrm{d}\left(\mathrm{d}_{1}, \mathrm{~d}_{2}\right), \mathrm{d}_{3}\right)= \\
=\mathrm{d}\left(\mathrm{d}_{1}, \mathrm{~d}\left(\mathrm{~d}_{2}, \mathrm{~d}_{3}\right)\right)\end{array}$ & $\begin{array}{l}\text { Агрегируются лишь частные оценки } \mathrm{d}_{\mathrm{j}} \text {, } \\
\text { принадлежащие одному уровню } \\
\text { иерархической структуры - «дерева» } \\
\text { качеств функционирования ВТС }\end{array}$ \\
\hline 3 & Гладкость & $\mathrm{d}\left(\mathrm{d}_{1}, \mathrm{~d}_{2}\right)$ - многочлен & $\begin{array}{l}\text { Это требование непрерывной зависимости } \\
\text { интегральной оценки от частных оценок }\end{array}$ \\
\hline 4 & Ограниченность & $\begin{array}{l}0 \leq \mathrm{d}\left(\mathrm{d}_{1}, \mathrm{~d}_{2}\right) \leq 1 \text { при } \\
0 \leq \mathrm{d}_{1}, \mathrm{~d}_{2} \leq 1\end{array}$ & $\begin{array}{l}\text { В квалиметрии задаются или } \\
\text { предполагаются известными границы } \\
\text { интервала изменения частных и } \\
\text { интегральной оценок }\end{array}$ \\
\hline 5 & Нейтральность & $\begin{array}{l}\mathrm{d}\left(\mathrm{d}_{1}, 0\right)=\mathrm{d}_{1}, \mathrm{~d}\left(0, \mathrm{~d}_{2}\right)=\mathrm{d}_{2} \\
\mathrm{~d}(0,0)=0, \mathrm{~d}(1,1)=1\end{array}$ & $\begin{array}{l}\text { Интегральная оценка совпадает с частной } \\
\text { оценкой, когда другая оценка принимает } \\
\text { минимальное значение }\end{array}$ \\
\hline
\end{tabular}

В работе [1] сформулирована следующая теорема. В классе многочленов двух переменных существует лишь три (с точностью до постоянных параметров) функции, удовлетворяющие условиям коммутативности и ассоциативности. Эти функции имеют вид:

1) $\mathrm{c}$;

2) $d_{1}+d_{2}+c$

3) $a\left(d_{1}+d_{2}\right)+b d_{1} d_{2}+a(a-1) / b$,

где $\mathrm{a}, \mathrm{b}, \mathrm{c}$ - произвольные константы, $\mathrm{b} \neq 0$.

Доказательство теоремы основано на представлении функции $\mathrm{d}\left(\mathrm{d}_{1}, \mathrm{~d}_{2}\right)$ в виде двойного степенного выражения с учетом, которое подставляется в условие ассоциативности. Учет аксиомы коммутативности и сравнение коэффициентов в обеих частях равенства приводят к функциям (1)-(3).

Оценка вида (1) тривиальна из-за независимости интегральной квалиметрической оценки от частных оценок. Вид (2) соответствует аддитивной свертке. Оценка вида 3) не может быть сведена к виду (1) или (2), так как $\mathrm{b} \neq 0$.

Следствие 1. Многочлен $\mathrm{f}\left(\mathrm{d}_{1}, \mathrm{~d}_{2}\right)$, удовлетворяющий свойствам ассоциативности, коммутативности и ограниченности (см. табл. 1), представим в виде

$$
\begin{aligned}
& \text { 1) } d_{1}+d_{2-} d_{1} d_{2} \text {; } \\
& \text { 2) } d_{1} d_{2} \text {. }
\end{aligned}
$$

Предположим, что $\mathrm{d}_{1}$ и $\mathrm{d}_{2} \square$ [q, $\left.\mathrm{Q}\right]$ и требуется, чтобы $\mathrm{d}\left(\mathrm{d}_{1}, \mathrm{~d}_{2}\right) \square[\mathrm{q}, \mathrm{Q}]$, причем $\mathrm{d}(\mathrm{q}, \mathrm{q})=\mathrm{q}$ и $\mathrm{d}(\mathrm{Q}, \mathrm{Q})=\mathrm{Q}$. 
Таблица 2. Основные виды ассоциативных интегральных квалиметрических оценок

\begin{tabular}{|c|c|c|c|c|c|}
\hline \multicolumn{4}{|c|}{ Аксиомы } & \multirow{2}{*}{$\begin{array}{c}\text { Вид интегральной } \\
\text { оценки } \mathrm{d}\left(\mathrm{d}_{1}, \mathrm{~d}_{2}\right)\end{array}$} & \multirow[b]{2}{*}{ Комментарии } \\
\hline $\begin{array}{c}\text { коммутатив- } \\
\text { ность }\end{array}$ & гладкость & $\begin{array}{c}\text { ограничен- } \\
\text { ность }\end{array}$ & $\begin{array}{c}\text { нейтраль- } \\
\text { ность }\end{array}$ & & \\
\hline+ & - & - & - & $\varphi^{-1}\left(\varphi\left(d_{1}\right)+\varphi\left(d_{2}\right)\right)$ & $\begin{array}{l}\varphi-\text { произвольная } \\
\text { строго монотонная } \\
\text { функция. Например, } \\
\text { при } \varphi(\mathrm{x})=\mathrm{x} \mathrm{и} \varphi(\mathrm{x})=\ln (\mathrm{x}) \\
\text { имеем аддитивную и } \\
\text { мультипликативную } \\
\text { интегральные оценки }\end{array}$ \\
\hline+ & - & - & + & $\varphi^{-1}\left(\varphi\left(d_{1}\right)+\varphi\left(d_{2}\right)\right)$ & $\begin{array}{l}\varphi-\text { монотонная } \\
\text { непрерывная } \\
\text { функция. Из аксиомы } \\
\text { нейтральности следует: } \\
\varphi\left(\mathrm{d}_{1}^{\mathrm{min}}\right)=\varphi\left(\mathrm{d}_{2}^{\mathrm{min}}\right)=0\end{array}$ \\
\hline+ & + & - & - & $\begin{array}{l}\text { 1) } \mathrm{c} \text {; } \\
\text { 2) } \mathrm{d}_{1}+\mathrm{d}_{2}+\mathrm{c} \\
\text { 3) } \mathrm{a}\left(\mathrm{d}_{1}+\mathrm{d}_{2}\right)+\mathrm{bd}_{1} \mathrm{~d}_{2}+ \\
\text { +a(a-1)/b }\end{array}$ & $\begin{array}{l}\mathrm{a}, \mathrm{b} \neq 0, \mathrm{c}- \\
\text { произвольные константы }\end{array}$ \\
\hline+ & + & + & - & $\begin{array}{l}\text { 1) } d_{1}+d_{2}-d_{1} d_{2} \\
\text { 2) } d_{1} d_{2}\end{array}$ & $\begin{array}{l}\text { Оценки } \mathrm{d}_{1}, \mathrm{~d}_{2} \text { и } \mathrm{d} \\
\text { принимают значения } \\
\text { из интервала [0,1]. } \\
\text { Интегральная оценка } \\
\text { 1) имеет смысл } \\
\text { риска недостижения } \\
\text { требуемого качества } \\
\text { функционирования } \\
\text { системы }\end{array}$ \\
\hline+ & + & + & + & $\mathrm{d}_{1}+\mathrm{d}_{2}-\mathrm{d}_{1} \mathrm{~d}_{2}$ & $\begin{array}{l}\text { Аналог вероятностной } \\
\text { свертки для } \\
\text { двух совместных } \\
\text { независимых событий }\end{array}$ \\
\hline
\end{tabular}

Запишем данные требования в виде системы уравнений

$$
\left\{\begin{array}{c}
f(q, q)=q \\
f(Q, Q)=Q
\end{array} .\right.
$$

С учетом общего вида коммутативного, ассоциативного многочлена, полученного из теоремы, система примет следующий вид:

$$
\left\{\begin{array}{c}
2 \mathrm{aq}+\mathrm{bq}^{2}+\mathrm{a}(\mathrm{a}-1) / \mathrm{b}=\mathrm{q} \\
2 \mathrm{aQ}+\mathrm{bQ}^{2}+\mathrm{a}(\mathrm{a}-1) / \mathrm{b}=\mathrm{Q}
\end{array} .\right.
$$

Из данной системы получаем следующее уравнение относительно а:

$$
2 \mathrm{a}(\mathrm{Q}-\mathrm{q})+\mathrm{b}(\mathrm{Q}-\mathrm{q})(\mathrm{Q}+\mathrm{q})=\mathrm{Q}-\mathrm{q} .
$$

Поскольку $\mathrm{Q}-\mathrm{q} \neq 0$, то $2 a+b(Q+q)=1$. Следовательно,

$$
\mathrm{a}=[1-\mathrm{b}(\mathrm{Q}+\mathrm{q})] / 2 \text {. }
$$


Подставим значение а, выраженное через b, в первое уравнение системы, получим:

$$
q[1-b(Q+q)]+b q^{2}-\left[1-b^{2}(Q+q)^{2}\right] / 4 b=q .
$$

Выполнив преобразования, получим следующее уравнение: $\mathrm{b}^{2}(\mathrm{Q}-\mathrm{q})^{2}=1$, откуда и $\mathrm{b}= \pm 1 /(\mathrm{Q}-\mathrm{q})$. Подставив значения b в выражение (4), получим соответствующие значения а. Таким образом, найдены два набора коэффициентов а и $b: a^{\prime}=-q /(Q-q), b^{\prime}=1 /(Q-q)$ и a" $=\mathrm{Q} /(\mathrm{Q}-\mathrm{q})$, $\mathrm{b}^{\prime \prime}=-1 /(\mathrm{Q}-\mathrm{q})$, а следовательно, и два вида зависимости $\mathrm{f}\left(\mathrm{d}_{1}, \mathrm{~d}_{2}\right)$ :

$$
\begin{aligned}
& \mathrm{d}^{\prime}=\mathrm{a}^{\prime}\left(\mathrm{d}_{1}+\mathrm{d}_{2}\right)+\mathrm{b}^{\prime} \mathrm{d}_{1} \mathrm{~d}_{2}+\mathrm{a}^{\prime}\left(\mathrm{a}^{\prime}-1\right) / \mathrm{b}^{\prime}=[-\mathrm{q} /(\mathrm{Q}-\mathrm{q})]\left(\mathrm{d}_{1}+\mathrm{d}_{2}\right)+[1 /(\mathrm{Q}-\mathrm{q})] \mathrm{d}_{1} \mathrm{~d}_{2}+ \\
& +[-\mathrm{q} /(\mathrm{Q}-\mathrm{q})][-\mathrm{q} /(\mathrm{Q}-\mathrm{q})-1] /[1 /(\mathrm{Q}-\mathrm{q})]= \\
& =1 /(\mathrm{Q}-\mathrm{q})\left[-\mathrm{q}\left(\mathrm{d}_{1}+\mathrm{d}_{2}\right)+\mathrm{d}_{1} \mathrm{~d}_{2}+\mathrm{Qq}\right], \\
& \mathrm{d}^{\prime \prime}=\mathrm{a}^{\prime \prime}\left(\mathrm{d}_{1}+\mathrm{d}_{2}\right)+\mathrm{b}^{\prime \prime} \mathrm{d}_{1} \mathrm{~d}_{2}+\mathrm{a}^{\prime \prime}\left(\mathrm{a}^{\prime \prime}-1\right) / \mathrm{b}^{\prime \prime}=[\mathrm{Q} /(\mathrm{Q}-\mathrm{q})]\left(\mathrm{d}_{1}+\mathrm{d}_{2}\right)+ \\
& +[-1 /(\mathrm{Q}-\mathrm{q})] \mathrm{d}_{1} \mathrm{~d}_{2}+[\mathrm{Q} /(\mathrm{Q}-\mathrm{q})][\mathrm{Q} /(\mathrm{Q}-\mathrm{q})-1] /[-1 /(\mathrm{Q}-\mathrm{q})]= \\
& =1 /(\mathrm{Q}-\mathrm{q})\left[\mathrm{Q}\left(\mathrm{d}_{1}+\mathrm{d}_{2}\right)-\mathrm{d}_{1} \mathrm{~d}_{2}+\mathrm{Q}\right] .
\end{aligned}
$$

Если $\mathrm{q}=0$ и $\mathrm{Q}=1$, то $\mathrm{d}_{1}, \mathrm{~d}_{2}, \mathrm{f}\left(\mathrm{d}_{1}, \mathrm{~d}_{2}\right) \square[0,1]$. В этом случае $\mathrm{d}^{\prime}=\mathrm{d}_{1} \mathrm{~d}_{2}, \mathrm{~d}^{\prime \prime}=\mathrm{d}_{1}+\mathrm{d}_{2}-\mathrm{d}_{1} \mathrm{~d}_{2}$. Что и требовалось показать.

Следствие 2. Многочлен $\mathrm{f}\left(\mathrm{d}_{1}, \mathrm{~d}_{2}\right)$, удовлетворяющий свойствам ассоциативности, коммутативности, ограниченности и нейтральности (см. табл. 1), представим в виде

$$
\mathrm{d}=\mathrm{d}_{1}+\mathrm{d}_{2}-\mathrm{d}_{1} \mathrm{~d}_{2}=1-\left(1-\mathrm{d}_{1}\right)\left(1-\mathrm{d}_{2}\right) \text {. }
$$

Отметим, что формула (5) совпадает с формулой вероятности суммы двух совместных независимых событий. (Вероятность суммы двух совместных событий А и В вычисляется по формуле $\mathrm{P}(\mathrm{A}+\mathrm{B})=\mathrm{P}(\mathrm{A})+\mathrm{P}(\mathrm{B})-\mathrm{P}(\mathrm{AB})$, a $\mathrm{P}(\mathrm{AB})=\mathrm{P}(\mathrm{A}) \mathrm{P}(\mathrm{B})$ для независимых событий.

Для построения интегральной квалиметрической оценки $\mathrm{d}$ нужно иметь частные относительные квалиметрические оценки $\mathrm{d}_{\mathrm{j}}$ функционирования ВТС по j-му ПК, j=1,2,..,m. Обозначим через $\mathrm{y}_{\mathrm{j}}^{\mathrm{i}}$ - значение $\mathrm{j}$-го ПК для і-го состояния ВТС, а через $\mathrm{y}_{\mathrm{j}}{ }^{*}-$ пороговое (предельно допустимое) значение, отражающее нормативное требование к качеству функционирования ВТС по j-му ПК. Поставим им в соответствие две безразмерные величины, принимающие значения из интервала $[0,1]: \mu_{\mathrm{j}}^{\mathrm{i}}=\mu_{\mathrm{j}}\left(\mathrm{y}_{\mathrm{j}}^{\mathrm{i}}\right)$ - абсолютную частную оценку качества и $\varepsilon_{\mathrm{j}}=\varepsilon_{\mathrm{j}}\left(\mathrm{y}_{\mathrm{j}}^{*}\right)_{-}$соответствующий нормативный уровень по ј-му ПК. Будем считать, что требование к качеству функционирования ВТС (например, требование к экологической безопасности района базирования) по ј-му ПК выполнено, если $\mu_{\mathrm{j}}^{\mathrm{i}} \geq \varepsilon_{\mathrm{j}}$. При этом частная оценка $\mathrm{d}_{\mathrm{j}}$, как функция величин $\varepsilon_{\mathrm{j}}$ и $\mu_{\mathrm{j}}$, должна удовлетворять следующим условиям: 1) $0 \leq \mathrm{d}_{\mathrm{j}} \leq 1$ при $\mu_{\mathrm{j}} \geq \varepsilon_{\mathrm{j}}$; 2) $\mathrm{d}_{\mathrm{j}}=0$ при $\varepsilon_{\mathrm{j}}=0, \mu_{\mathrm{j}}>0$ (оценка минимальна, если нет никаких требований к качеству); 3) $\mathrm{d}_{\mathrm{j}}=0$ при $\mu_{\mathrm{j}}=1$ и $\mu_{\mathrm{j}}>\varepsilon_{\mathrm{j}}$ (оценка минимальна при «идеальном» качестве независимо от требований); 4) $\mathrm{d}_{\mathrm{j}}=1$ при $\mu_{\mathrm{j}}=\varepsilon_{\mathrm{j}} \neq 0$ (оценка максимальна при предельно низком допустимом качестве). В работах [1-4] показано, что при $\mu_{\mathrm{j}} \geq \varepsilon_{\mathrm{j}}$ условиям 1)-4) удовлетворяет частная квалиметрическая оценка $\mathrm{d}_{\mathrm{j}}$ вида

$$
\mathrm{d}_{\mathrm{j}}=\left[\varepsilon_{\mathrm{j}}\left(1-\mu_{\mathrm{j}}\right)\right] /\left[\mu_{\mathrm{j}}\left(1-\varepsilon_{\mathrm{j}}\right)\right] .
$$

Покажем, что данная оценка позволяет вычислять условную вероятность события, состоящего в том, что требование к интегральному качеству функционирования ВТС не выпол- 
няется при выполнении требований к ее качеству по ј-му частному ПК. Обозначим через $\mu$ интегральное качество (качество в целом) функционирования ВТС, а через $\varepsilon$ - требование к этому качеству. Будем считать, что требование к интегральному качеству функционирования ВТС выполнено, если $\mu \geq \varepsilon$. Причем если качество $\mu_{\mathrm{j}}$ функционирования ВТС хотя бы по одному ПК ниже соответствующего нормативного уровня $\varepsilon_{\mathrm{j}}$, то удовлетворить требование к интегральному качеству функционирования ВТС невозможно. Пусть А - событие, состоящее в том, что не выполнено требование к интегральному качеству, а $\mathrm{B}_{\mathrm{j}}$ - событие, состоящее в том, что не выполнено требование к качеству функционирования ВТС по ј-му ПК. Тогда условная вероятность $\mathrm{P}\left(\mathrm{A} \mid \mathrm{B}_{\mathrm{j}}\right)=1$, так как если $\mu_{\mathrm{j}}<\varepsilon_{\mathrm{j}}$,то $\mu<\varepsilon$. В то же время $0 \leq \mathrm{P}\left(\mathrm{A} \mid \overline{\mathrm{B}}_{\mathrm{j}}\right) \leq 1$, где $\bar{B}_{j}-$ событие, противоположное $\mathrm{B}_{\mathrm{j}}$. Другими словами, $\mathrm{P}\left(\mathrm{A} \mid \overline{\mathrm{B}}_{\mathrm{j}}\right)$ - вероятность невыполнения требований к качеству функционирования ВТС при условии, что требования к качеству функционирования ВТС выполнены по ј-му ПК. Эту вероятность и нужно определить. Отметим, что события $\mathrm{AB}_{\mathrm{j}}$, и $A \bar{B}_{j}$ попарно несовместны и $A=A B_{j}+A \bar{B}_{j}$. Так как, $\mathrm{P}\left(\mathrm{A} \mid \mathrm{B}_{\mathrm{j}}\right)=1$, то из формулы полной вероятности следует $\mathrm{P}(\mathrm{A})=\mathrm{P}\left(\mathrm{AB}_{\mathrm{j}}\right)+\mathrm{P}\left(\overline{\mathrm{A}}_{\mathrm{j}}\right)=\mathrm{P}\left(\mathrm{B}_{\mathrm{j}}\right) \mathrm{P}\left(\mathrm{A} \mid \mathrm{B}_{\mathrm{j}}\right)+\mathrm{P}\left(\overline{\mathrm{B}}_{\mathrm{j}}\right) \mathrm{P}\left(\mathrm{A} \mid \overline{\mathrm{B}}_{\mathrm{j}}\right)=\mathrm{P}\left(\mathrm{B}_{\mathrm{j}}\right)+\left[1-\mathrm{P}\left(\mathrm{B}_{\mathrm{j}}\right)\right] \mathrm{P}\left(\mathrm{A} \mid \overline{\mathrm{B}}_{\mathrm{j}}\right)$. Таким образом, $\mathrm{P}\left(\mathrm{A} \mid \overline{\mathrm{B}}_{\mathrm{j}}\right)=\left[\mathrm{P}(\mathrm{A})-\mathrm{P}\left(\mathrm{B}_{\mathrm{j}}\right)\right] /\left[1-\mathrm{P}\left(\mathrm{B}_{\mathrm{j}}\right)\right]$. Рассмотрим формулу умножения вероятностей $\mathrm{P}\left(\mathrm{AB}_{\mathrm{j}}\right)=\mathrm{P}(\mathrm{A}) \mathrm{P}\left(\mathrm{B}_{\mathrm{j}} \mid \mathrm{A}\right)=\mathrm{P}\left(\mathrm{B}_{\mathrm{j}}\right) \mathrm{P}\left(\mathrm{A} \mid \mathrm{B}_{\mathrm{j}}\right)$. Опять учитывая, что $\mathrm{P}\left(\mathrm{A} \mid \mathrm{B}_{\mathrm{j}}\right)=1$, получаем важное неравенство $0 \leq \mathrm{P}(\mathrm{A})=\mathrm{P}\left(\mathrm{B}_{\mathrm{j}}\right) / \mathrm{P}\left(\mathrm{B}_{\mathrm{j}} \mid \mathrm{A}\right) \leq 1$. Отсюда следует, что $\mathrm{P}\left(\mathrm{B}_{\mathrm{j}}\right) \leq \mathrm{P}\left(\mathrm{B}_{\mathrm{j}} \mid \mathrm{A}\right)$. В результате получаем:

$$
\mathrm{P}\left(\mathrm{A} \mid \overline{\mathrm{B}}_{\mathrm{j}}\right)=\left\{\mathrm{P}\left(\mathrm{B}_{\mathrm{j}}\right)\left[1-\mathrm{P}\left(\mathrm{B}_{\mathrm{j}} \mid \mathrm{A}\right)\right]\right\} /\left\{\mathrm{P}\left(\mathrm{B}_{\mathrm{j}} \mid \mathrm{A}\right)\left[1-\mathrm{P}\left(\mathrm{B}_{\mathrm{j}}\right)\right]\right\} \text {. }
$$

Введем следующие обозначения: $\mathrm{d}_{\mathrm{j}}=\mathrm{P}\left(\mathrm{A} \mid \overline{\mathrm{B}}_{\mathrm{j}}\right), \varepsilon_{\mathrm{j}}=\mathrm{P}\left(\mathrm{B}_{\mathrm{j}}\right)$ - вероятность некачественности функционирования ВТС по ј-му ПК, $\mu_{\mathrm{j}}=\mathrm{P}\left(\mathrm{B}_{\mathrm{j}} \mid \mathrm{A}\right)$ - вероятность некачественности функционирования ВТС по ј-му ПК при условии, что требования к качеству функционирования ВТС не выполнены. В результате получаем, что формула (7) совпадает с формулой (6) частной относительной оценки $\mathrm{d}_{\mathrm{j}}$ типа «риск недостижения требуемого качества функционирования ВТС», которая является квалиметрической моделью частной относительной оценки опасности функционирования ВТС. В силу вероятностной природы величин $\mathrm{d}_{\mathrm{j}}, \varepsilon_{\mathrm{j}}, \mu_{\mathrm{j}}$ они принимают значения из интервала $[0,1]$. Причем, $\mu_{\mathrm{j}} \geq \varepsilon_{\mathrm{j}}$.

Доопределим оценку $\mathrm{d}_{\mathrm{j}}$ следующим образом: 1) $\mathrm{d}_{\mathrm{j}}=1$ при $\mu_{\mathrm{j}}=\varepsilon_{\mathrm{j}}=1$; 2) $\mathrm{d}_{\mathrm{j}}=0$ при $\mu_{\mathrm{j}}=\varepsilon_{\mathrm{j}}=0$; 3) $\mathrm{d}_{\mathrm{j}}=1$ для всех $\mu_{\mathrm{j}}<\varepsilon_{\mathrm{j}}$. Отметим, что оценка $\mathrm{d}_{\mathrm{j}}$ является убывающей функцией по $\mu_{\mathrm{j}}$ и возрастающей по $\varepsilon_{j}$.

В общем случае для $\mathrm{m}$ частных оценок интегральная квалиметрическая оценка функционирования ВТС имеет следующую структуру [1]:

$$
\mathrm{d}=1-\prod_{\mathrm{j}=1}^{\mathrm{m}}\left(1-\mathrm{d}_{\mathrm{j}}\right) .
$$

Операцию, задаваемую функцией вида (1), будем называть обобщенным сложением (квазисложением) и обозначать символом $\oplus: \mathrm{d}=\mathrm{d}_{1} \oplus \mathrm{d}_{2}$. Эта операция обладает всеми свойствами обычной операции сложения. Можно показать [1], что операция обобщенного умножения на произвольное неотрицательное число $\lambda$, которую обозначим символом $\otimes$, может быть введена в форме

$$
\lambda_{\mathrm{j}} \otimes \mathrm{d}_{\mathrm{j}}=1-\left(1-\mathrm{d}_{\mathrm{j}}\right)^{\lambda \mathrm{j}}
$$


Данная операция согласована с операцией квазисложения и удовлетворяет соотношениям вида: $1 \otimes \mathrm{d}_{\mathrm{j}}=\mathrm{d}_{\mathrm{j}}, \lambda_{0} \otimes\left(\mathrm{d}_{1} \oplus \mathrm{d}_{2}\right)=\lambda_{0} \otimes \mathrm{d}_{1} \oplus \lambda_{0} \otimes \mathrm{d}_{2},\left(\lambda_{1} \oplus \lambda_{2}\right) \otimes \mathrm{d}_{\mathrm{j}}=\lambda_{1} \otimes \mathrm{d}_{\mathrm{j}} \oplus \lambda_{2} \otimes \mathrm{d}_{\mathrm{j}}$.

Подчеркнем, что формула (8) интегральной квалиметрической оценки получена в предположении коммутативности (равноценности) и ассоциативности (иерархической одноуровненности) частных оценок. Использование весовых коэффициентов $\lambda_{\mathrm{j}}$ для «выравнивания» значимости частных квалиметрических оценок $\mathrm{d}_{\mathrm{j}}$ само по себе противоречиво. Аксиома коммутативности уже предполагает равноценность этих оценок. Такого рода «равноправность» достигается единым способом измерения частных абсолютных оценок качества $\mu_{\mathrm{j}}$ и нормативных уровней $\varepsilon_{j}$.

Поэтому некоммутативная аддитивная линейная свертка оценок $\mathrm{d}_{1}$ и $\mathrm{d}_{2}$ вида $\lambda_{1} \mathrm{~d}_{1}+\lambda_{2} \mathrm{~d}_{2}$ становится коммутативной для величин $\mathrm{d}_{1}^{\prime}=\lambda_{1} \mathrm{~d}_{1}$ и $\mathrm{d}_{2}=\lambda_{2} \mathrm{~d}_{2}$. Рассмотрим конкретные способы «выравнивания» частных оценок $\mathrm{d}_{\mathrm{j}}$.

Из формул (6) и (9) для $\lambda_{\mathrm{j}}>0$ имеем:

$$
\left[\varepsilon_{\mathrm{j}}^{\prime}\left(1-\mu_{\mathrm{j}}^{\prime}\right)\right] /\left[\mu_{\mathrm{j}}^{\prime}\left(1-\varepsilon_{\mathrm{j}}^{\prime}\right)\right]=.\left(\varepsilon_{\mathrm{j}}^{\prime} / \mu_{\mathrm{j}}^{\prime}-\varepsilon_{\mathrm{j}}^{\prime}\right) /\left(1-\varepsilon_{\mathrm{j}}^{\prime}\right)=\mathrm{d}_{\mathrm{j}}^{\prime}=\lambda_{\mathrm{j}} \otimes \mathrm{d}_{\mathrm{j}}=1-\left(1-\mathrm{d}_{\mathrm{j}}\right)^{\lambda_{\mathrm{j}}} .
$$

Покажем, что здесь $\varepsilon_{\mathrm{j}}^{\prime}=1-\left(1-\varepsilon_{\mathrm{j}}\right)^{\lambda_{\mathrm{j}}}, \mu_{\mathrm{j}}^{\prime}=\left[1-\left(1-\varepsilon_{\mathrm{j}}\right)^{\lambda_{\mathrm{j}}}\right] /\left[1-\left(1-\varepsilon_{\mathrm{j}} / \mu_{\mathrm{j}}\right)^{\lambda_{j}}\right]$. Действительно,

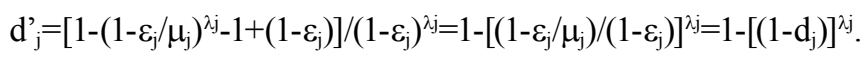

Таким образом, в общем случае интегральной квалиметрической оценке типа «общий риск недостижения требуемого качества функционирования ВТС» соответствует квалиметрическая модель вида

$$
\mathrm{d}=1-\prod_{\mathrm{j}=1}^{\mathrm{m}}\left(1-\mathrm{d}_{\mathrm{j}}\right)^{\lambda_{\mathrm{j}}}
$$

где $\lambda_{\mathrm{j}}$ - весовые коэффициенты частных оценок $\mathrm{d}_{\mathrm{j}}$, удовлетворяющие условию нормировки:

$$
\sum_{j=1}^{m} \lambda_{\mathrm{j}}=1, \lambda_{\mathrm{j}} \geq 0, \mathrm{j}=1,2, \ldots, \mathrm{m}
$$

Подчеркнем, что интегральная квалиметрическая оценка d в формуле (10) при $\lambda_{\mathrm{j}}=1 / \mathrm{m}$ является средней величиной в смысле ассоциативной средней по Колмогорову [5], которая вычисляется по формуле

$$
\mathrm{d}\left(\mathrm{d}_{1}, \mathrm{~d}_{2}, \ldots, \mathrm{d}_{\mathrm{m}}\right)=\varphi^{-1}\left(\frac{1}{\mathrm{~m}} \varphi\left(\mathrm{d}_{1}\right)+\frac{1}{\mathrm{~m}} \varphi\left(\mathrm{d}_{2}\right)+\ldots+\frac{1}{\mathrm{~m}} \varphi\left(\mathrm{d}_{\mathrm{m}}\right)\right) .
$$

Здесь $\varphi$ - непрерывная строго монотонная функция, а $\varphi^{-1}-$ функция, обратная к ней. В нашем случае $\varphi\left(\mathrm{d}_{\mathrm{j}}\right)=-\ln \left(1-\mathrm{d}_{\mathrm{j}}\right)$. При $\varphi\left(\mathrm{d}_{\mathrm{j}}\right)=\ln \mathrm{d}_{\mathrm{j}}$ имеем среднюю геометрическую. Так, в работе Э. Харрингтона [6] частными оценками качества системы являются экспоненциальные функции «желательности», а интегральная оценка есть среднее геометрическое этих частных оценок. В то же время средняя квазигеометрическая величина $\mathrm{d}$ в формуле (8) не средняя величина по Коши [5], т.е. не удовлетворяет условию $\min \left(\mathrm{d}_{1}, \mathrm{~d}_{2}\right) \leq \mathrm{d} \leq \max \left(\mathrm{d}_{1}, \mathrm{~d}_{2}\right)$. Покажем, что средневзвешенное квазигеометрическое есть среднее по Коши. В силу коммутативности квалиме- 
трической оценки $\mathrm{d}$ рассмотрим только случай $\mathrm{d}_{1} \geq \mathrm{d}_{2}$. Так как $\min \left(\mathrm{d}_{1}, \mathrm{~d}_{2}\right)=\mathrm{d}_{2}, \max \left(\mathrm{d}_{1}, \mathrm{~d}_{2}\right)=\mathrm{d}_{1}$ то имеем:

$$
\begin{aligned}
& 1-\left(1-\mathrm{d}_{1}\right)^{\lambda_{1}}\left(1-\mathrm{d}_{2}\right)^{\lambda_{2}} \leq 1-\left(1-\mathrm{d}_{1}\right)^{\lambda_{1}}\left(1-\mathrm{d}_{1}\right)^{\lambda_{2}}=\mathrm{d}_{1}=\max \left(\mathrm{d}_{1}, \mathrm{~d}_{2}\right), \\
& 1-\left(1-\mathrm{d}_{1}\right)^{\lambda_{1}}\left(1-\mathrm{d}_{2}\right)^{\lambda_{2}} \geq 1-\left(1-\mathrm{d}_{2}\right)^{\lambda_{1}}\left(1-\mathrm{d}_{2}\right)^{\lambda_{2}}=\mathrm{d}_{2}=\min \left(\mathrm{d}_{1}, \mathrm{~d}_{2}\right) .
\end{aligned}
$$

Таким образом, $\min \left(\mathrm{d}_{1}, \mathrm{~d}_{2}\right) \leq 1-\left(1-\mathrm{d}_{1}\right)^{\lambda_{1}}\left(1-\mathrm{d}_{2}\right)^{\lambda_{2}} \leq \max \left(\mathrm{d}_{1}, \mathrm{~d}_{2}\right)$ и средневзвешенное квазигеометрическое $\mathrm{d}$ есть среднее по Коши.

Приведем геометрическую интерпретацию квалиметрических оценок. «Отрезок» $\overline{\mathrm{d}}=\lambda \otimes \overline{\mathrm{d}}^{1} \oplus(1-\lambda) \otimes \overline{\mathrm{d}}^{2} \quad(0 \leq \lambda \leq 1)$, соединяющий две точки $\bar{d}^{1}=\left(\mathrm{d}_{1}{ }_{1}, \mathrm{~d}^{1}{ }_{2}\right)$ и $\bar{d}^{2}=\left(\mathrm{d}^{2}{ }_{1}, \mathrm{~d}^{2}{ }_{2}\right)$ в пространстве частных квалиметрических оценок, показан на рис. 1. Средневзвешенной арифметической величине соответствует отрезок прямой $\overline{\mathrm{d}}_{\mathrm{a}}=\lambda \overline{\mathrm{d}}^{1}+(1-\lambda) \overline{\mathrm{d}}^{2}$, а средневзвешенной геометрической - «отрезок» $\overline{\mathrm{d}}_{\mathrm{g}}=\left(\overline{\mathrm{d}}^{1}\right)^{\lambda}\left(\overline{\mathrm{d}}^{2}\right)^{1-\lambda}$.

Подчеркнем, что с теоретико-информационной точки зрения $\left[7, \mathrm{c.} \mathrm{38]} \mathrm{число} \varphi\left(\mathrm{d}_{\mathrm{j}}\right)=-\ln \left(1-\mathrm{d}_{\mathrm{j}}\right)\right.$ является мерой неопределенности информации (частной информационной оценкой) при вычислении энтропии события, так как частную квалиметрическую оценку $\mathrm{d}_{\mathrm{j}}$ можно интерпретировать как вероятность $\mathrm{P}\left(\mathrm{A} \mid \overline{\mathrm{B}}_{\mathrm{j}}\right)$ события $A$, состоящего в том, что не выполнено требование к интегральному качеству функционирования ВТС, при условии, что выполняется событие $\overline{\mathrm{B}}_{\mathrm{j}}$, состоящее в том, что требование к качеству функционирования ВТС по ј-му ПК выполнено [1]. Таким образом, событию $\left(\mathrm{A} \mid \overline{\mathrm{B}}_{\mathrm{j}}\right)$ соответствует неопределенность, равная $-\ln \left[\mathrm{P}\left(A \mid \bar{B}_{j}\right)\right]=$ $\ln \left[1 / \mathrm{P}\left(\mathrm{A} \mid \overline{\mathrm{B}}_{\mathrm{j}}\right)\right]$, а противоположному событию $\left(\overline{\mathrm{A}} \mid \overline{\mathrm{B}}_{\mathrm{j}}\right)$ - частная информационная оценка $\mathrm{I}_{\mathrm{j}}=\mathrm{I}_{\mathrm{j}}\left(\mathrm{d}_{\mathrm{j}}\right)=$ $-\ln \left[\mathrm{P}\left(\bar{A} \mid \bar{B}_{j}\right)\right]=\ln \left[1 /\left(1-\mathrm{P}\left(A \mid \bar{B}_{j}\right)\right]=\ln \left[1 /\left(1-\mathrm{d}_{\mathrm{j}}\right)\right]\right.$. Укажем основные свойства оценок $\left.\mathrm{I}_{\mathrm{j}}: 1\right) \mathrm{I}_{\mathrm{j}}=0$ при $\mathrm{d}_{\mathrm{j}}=0$; 2) $\mathrm{I}_{\mathrm{j}} \rightarrow \infty$ при $\mathrm{d}_{\mathrm{j}} \rightarrow 1$.

Отметим, что интегральная информационная оценка $\mathrm{I}=\mathrm{I}(\mathrm{d})=\ln [1 /(1-\mathrm{d})]=\sum_{\mathrm{j}=1}^{\mathrm{m}} \lambda_{\mathrm{j}} \mathrm{I}_{\mathrm{j}}$ есть средневзвешенная арифметическая величина, которая является функцией средневзвешенного «квазигеометрического» - интегральной квалиметрической оценки $\mathrm{d}$, соответствующей формуле (10). Можно показать, что величина $\mathrm{I}=-\ln (1-\mathrm{d})$ - «квазисредняя» величина

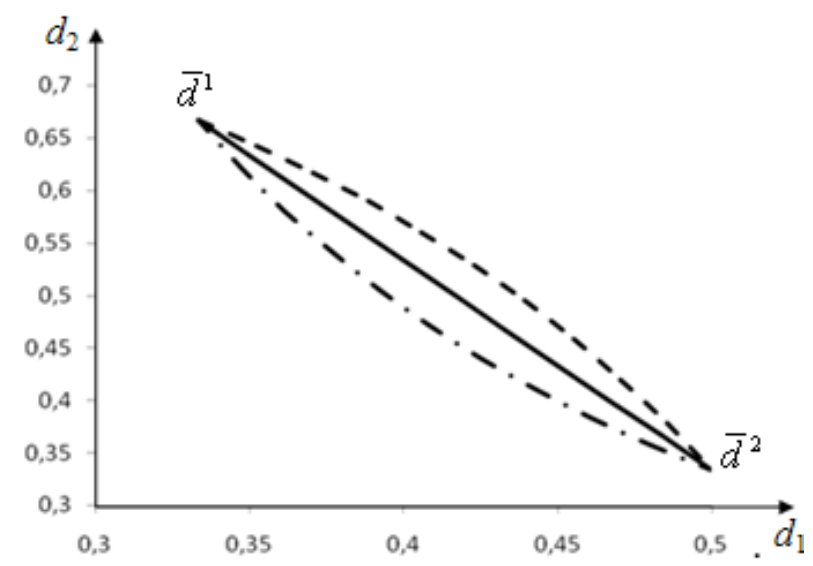

Рис. 1. Графическое представление средневзвешенных величин: _ _ _ _ _ - квазигеометрическое; - арифметическое; - геометрическое 
(длина в натурально-логарифмической шкале), обеспечивающая минимум «квазидисперсии»:

$$
\mathrm{S}_{\mathrm{d}}=\sum_{\mathrm{j}=1}^{\mathrm{m}} \lambda_{\mathrm{j}}\left(\ln \frac{1}{1-\mathrm{d}_{\mathrm{j}}}-\ln \frac{1}{1-\mathrm{d}}\right)^{2}=\sum_{\mathrm{j}=1}^{\mathrm{m}} \lambda_{\mathrm{j}}\left[\ln \left(1-\mathrm{d}_{\mathrm{j}}\right)-\ln (1-\mathrm{d})\right]^{2} .
$$

Для двух частных квалиметрических оценок $\mathrm{d}_{1}$ и $\mathrm{d}_{2}$ с весовыми коэффициентами $\lambda_{1}$ и $\lambda_{2}$ соответственно имеем:

$$
\begin{aligned}
& S_{d}=\lambda_{1}\left(\ln \left(1-d_{1}\right)-\ln (1-d)\right)^{2}+\lambda_{2}\left(\ln \left(1-d_{2}\right)-\ln (1-d)\right)^{2}= \\
& =\left(\lambda_{1}+\lambda_{2}\right)[\ln (1-d)]^{2}-2\left(\lambda_{1} \ln \left(1-d_{1}\right)+\lambda_{2} \ln \left(1-d_{2}\right)\right) \ln (1-d)+\lambda_{1}\left(\ln \left(1-d_{1}\right)\right)^{2}+\lambda_{2}\left(\ln \left(1-d_{2}\right)\right)^{2} .
\end{aligned}
$$

Из условия оптимальности следует, что $\ln (1-\mathrm{d})=\left(\lambda_{1} \ln \left(1-\mathrm{d}_{1}\right)+\lambda_{2} \ln \left(1-\mathrm{d}_{2}\right)\right) /\left(\lambda_{1}+\lambda_{2}\right)$. Значит, $\mathrm{d}=1-\left(1-\mathrm{d}_{1}\right)^{\lambda 1}\left(1-\mathrm{d}_{2}\right)^{\lambda 2}$. В общем случае для m частных оценок $\mathrm{d}_{\mathrm{j}}$ получаем формулу $(10)$.

На этом этапе формирование интегральной квалиметрической оценки можно было бы считать завершенным, но обычно полученные величины I модифицируются. Дело в том, что частная квалиметрическая оценка $\mathrm{d}_{\mathrm{j}}$ и частная информационная оценка $\mathrm{I}_{\mathrm{j}}$ устроены так, что и «улучшение» качества функционирования ВТС совпадает с уменьшением значений $\mathrm{d}_{\mathrm{j}}$ и $\mathrm{I}_{\mathrm{j}}$. Традиционно принят обратный («зеркальный») счет. Зададимся некоторой величиной $\mathrm{d}_{\mathrm{e}}$ такой, что

$$
\overline{\mathrm{I}}_{\mathrm{j}}=\ln \frac{1}{1-\mathrm{d}_{\mathrm{e}}}-\ln \frac{1}{1-\mathrm{d}_{\mathrm{j}}}=\ln \frac{1-\mathrm{d}_{\mathrm{j}}}{1-\mathrm{d}_{\mathrm{e}}}=\ln \frac{1}{1-\mathrm{d}_{\mathrm{e}}}-\mathrm{I}_{\mathrm{j}} .
$$

Если за $\mathrm{d}_{\mathrm{e}}$ взять величину $\mathrm{d}_{\mathrm{e}}=1-\frac{1}{\mathrm{e}} \approx 0.63$, то $\ln \frac{1}{1-d_{e}}=1$, a $\overline{\mathrm{I}}_{\mathrm{j}}=1+\ln \left(1-\mathrm{d}_{\mathrm{j}}\right)$, тогда $\overline{\mathrm{I}}=\sum_{\mathrm{j}=1}^{\mathrm{m}} \lambda_{\mathrm{j}}+\sum_{\mathrm{j}=1}^{\mathrm{m}} \lambda_{\mathrm{j}} \ln \left(1-\mathrm{d}_{\mathrm{j}}\right)=1+\sum_{\mathrm{j}=1}^{\mathrm{m}} \lambda_{\mathrm{j}} \ln \left(1-\mathrm{d}_{\mathrm{j}}\right)=1-\sum_{\mathrm{j}=1}^{\mathrm{m}} \lambda_{\mathrm{j}} \ln \frac{1}{1-\mathrm{d}_{\mathrm{j}}}=1-\sum_{\mathrm{j}=1}^{\mathrm{m}} \lambda_{\mathrm{j}} \mathrm{I}_{\mathrm{j}}=1-\mathrm{I}$.

Для содержательной интерпретации квалиметрических оценок функционирования ВТС предлагается использовать зеркальную вербально-числовую шкалу Харрингтона [5], для которой величина $\mathrm{d}_{\mathrm{e}}=1-1 / \mathrm{e} \approx 0.63$ является особой точкой - точкой перехода системы в «некачественное» состояние.

Если величины $d_{j}$ рассматривать как риски (вероятности) недостижения требуемого качества функционирования BТC по j-му ПК (j-му потребляемому ресурсу), то оценки $\mathrm{I}\left(\mathrm{d}_{\mathrm{j}}\right)$ отражают информацию о значимости ПК (о степени дефицитности ресурсов по качеству). Причем чем больше значение $\mathrm{I}\left(\mathrm{d}_{\mathrm{j}}\right)$, тем больше чувствительность управленческих решений к изменению квалиметрической оценки $\mathrm{d}_{\mathrm{j}}$ (изменению качества ј-го ресурса) для достижения качества функционирования ВТС. Это позволяет рассматривать $\mathrm{I}\left(\mathrm{d}_{\mathrm{j}}\right)$ как своего рода универсальные стоимостные измерители единицы ј-го ресурса и использовать их в роли коэффициентов информационной линейной целевой функции типа обобщенных затрат в моделях многокритериальной ресурсной оптимизации сложной системы [1]. Данная целевая функция стимулирует эффективное использование дефицитных по качеству ресурсов в процессе функционирования ВТС.

Покажем, что интегральные оценки di можно применять в системах мониторинга управления функционированием, а именно для оценки изменений качества функционирования ВТС с учетом дополнительной информации (наблюдений) о состоянии «новых» систем. Пусть сред- 
ствами мониторинга получены $\mathrm{L}$ «новых» наблюдений, которым соответствуют значения $\mathrm{y}_{\mathrm{j}}^{\mathrm{i}}$ показателей качества, $\mathrm{j}=1,2, \ldots, \mathrm{m} ; \mathrm{i}=\mathrm{N}+1, \mathrm{~N}+2, \ldots, \mathrm{N}+\mathrm{L}$. Таким образом, к первоначальному списку $\mathrm{N}$ состояний систем добавилось $\mathrm{L}$ «новых» с тем же набором ПК (по наименованиям и количеству). Необходимо упорядочить весь имеющийся список $\mathrm{N}+\mathrm{L}$ систем по уровню качества функционирования с учётом ранжировки первоначального списка ВТС. Если в «первоначальном» ранжировании одна ВТС лучше другой (или они эквивалентны) в смысле качества функционирования, то добавление новых систем не должно изменить уже имеющееся отношение предпочтительности (эквивалентности). Таким образом, требуется определить интегральные квалиметрические оценки с учетом новой информации о состоянии функционирования ВТС на фоне ранжировки первоначальных систем.

Будем считать, что с появлением новой информации может изменяться исходная экспертная информация, необходимая для построения интегральной квалиметрической оценки. А именно:

1) заданы новые интервалы изменения ј-го ПК - $\left[\mathrm{A}_{\mathrm{j}}, \mathrm{B}_{\mathrm{j}}\right]$, где $\mathrm{A}_{\mathrm{j}} \leq \mathrm{a}_{\mathrm{j}}$ и $\mathrm{B}_{\mathrm{j}} \geq \mathrm{b}_{\mathrm{j}}$;

2) заданы новые предельно допустимые значения и новые нормативные уровни $-Y_{j}^{*}, E_{j}$.

Предполагается, что для каждого ПК остаются без изменения способ нормировки (см. табл. 2) и значения весовых коэффициентов $\lambda_{\mathrm{j}}$ частных оценок $\mathrm{d}_{\mathrm{j}}$. Для всего списка $(\mathrm{N}+\mathrm{L})$ систем с учетом новой информации частная абсолютная квалиметрическая оценка $\mathrm{M}_{\mathrm{j}}^{\mathrm{i}}$ по $\mathrm{j}$-му ПК для i-го состояния ВТС имеет вид, аналогичный виду $\mu_{\mathrm{j}}^{\mathrm{i}}$ (см. табл. 2): $\mathrm{M}_{\mathrm{j}}^{\mathrm{i}}=\mathrm{M}_{\mathrm{j}}^{\mathrm{i}}\left(\mathrm{y}_{\mathrm{j}}^{\mathrm{i}}, \mathrm{A}_{\mathrm{j}} \mathrm{B}_{\mathrm{j}}\right.$ ). Частная относительная $\mathrm{D}_{\mathrm{j}}^{\mathrm{i}}$ по $\mathrm{j}$-му ПК и интегральная $\mathrm{D}^{\mathrm{i}}$ квалиметрические оценки для i-го состояния ВТС вычисляются по формулам

$$
\begin{aligned}
& D_{j}^{i}=\left[E_{j}\left(1-M_{j}^{i}\right)\right] /\left[M_{j}^{i}\left(1-E_{j}\right)\right], \\
& D^{i}=1-\prod_{j=1}^{m}\left(1-D_{j}^{i}\right)^{\lambda_{j}}, i=1,2, \ldots, N+L ; j=1,2, \ldots, m .
\end{aligned}
$$

При этом ранжировки первоначального списка $\mathrm{N}$ систем по значениям $\mathrm{D}^{\mathrm{i}}$ и $\mathrm{d}^{\mathrm{i}}$ будут отличаться друг от друга.

Утверждение. Существуют преобразования квалиметрических оценок, которые позволяют произвести упорядочение всех анализируемых систем, сохраняющее отношения предпочтительности (эквивалентности) в первоначальном списке при получении новой информации.

Доказательство. Для включения новых ВТС в ранжировку первоначального списка по значениям $\mathrm{d}^{\mathrm{i}}$ необходимо «скорректировать» значения величин $\mathrm{M}_{\mathrm{j}}^{\mathrm{i}} \mathrm{c}$ помощью преобразования $\hat{M}_{j}^{i}=F\left(M_{j}^{i}\right)$. При этом значения частной относительной $\hat{D}_{j}^{i}$ и интегральной $\hat{D}^{i}$ квалиметрических оценок вычисляются по формулам

$$
\begin{aligned}
& \hat{D}_{j}^{i}=\left[E_{j}\left(1-\hat{M}_{j}^{i}\right)\right] /\left[\hat{M}_{j}^{i}\left(1-E_{j}\right)\right], \\
& \hat{D}^{i}=1-\prod_{j=1}^{m}\left(1-\hat{D}_{j}^{i}\right)^{\lambda_{j}} .
\end{aligned}
$$

Эти оценки обладают следующими свойствами: 1) если $\mathrm{d}^{\mathrm{i}}>\mathrm{d}^{1}$, то и $\hat{\mathrm{D}}^{\mathrm{i}}>\hat{\mathrm{D}}^{1}$;2) если $\mathrm{d}^{\mathrm{i}}=\mathrm{d}^{1}$, то и $\hat{\mathrm{D}}^{\mathrm{i}}=\hat{\mathrm{D}}^{1}, \mathrm{i}, \mathrm{l}=1,2, \ldots, \mathrm{N}$.

Неравенство $\mathrm{d}^{\mathrm{i}} \geq \mathrm{d}^{1}$ перепишем в виде 


$$
1-\prod_{\mathrm{j}=1}^{\mathrm{m}}\left(1-\mathrm{d}_{\mathrm{j}}^{\mathrm{i}}\right)^{\lambda_{\mathrm{j}}} \geq 1-\prod_{\mathrm{j}=1}^{\mathrm{m}}\left(1-\mathrm{d}_{\mathrm{j}}^{1}\right)^{\lambda_{\mathrm{j}}}
$$

Вначале рассмотрим случай, когда изменение состояния системы рассматривается лишь с точки зрения «корректировки» одной частной квалиметрической оценки $\mathrm{d}_{\mathrm{j}}$. Без ограничения общности можно считать, что это оценка $\mathrm{d}_{1}$. Тогда неравенство (7) перепишется следующим образом:

$$
\left(1-d_{1}^{1}\right)^{\lambda_{1}} \geq\left(1-d_{1}^{i}\right)^{\lambda_{1}}\left[\prod_{j=2}^{m}\left(1-d_{j}^{i}\right)^{\lambda_{j}} / \prod_{j=2}^{m}\left(1-d_{j}^{1}\right)^{\lambda_{j}}\right]
$$

Введем в рассмотрение величину $\quad \mathrm{D}^{\mathrm{il}}=\prod_{\mathrm{j}=2}^{\mathrm{m}}\left(\left[1-\mathrm{d}_{\mathrm{j}}^{\mathrm{i}}\right] /\left[1-\mathrm{d}_{\mathrm{j}}^{1}\right]\right)^{\frac{\lambda_{\mathrm{i}}}{\lambda_{1}}} . \quad$ Поскольку $1-\mathrm{d}_{\mathrm{j}}^{\mathrm{i}}=1-\left[\varepsilon_{\mathrm{j}}\left(1-\mu_{\mathrm{j}}^{\mathrm{i}}\right)\right] /\left[\left(1-\varepsilon_{\mathrm{j}}\right) \mu_{\mathrm{j}}^{\mathrm{i}}\right]=\left[\mu_{\mathrm{j}}^{\mathrm{i}}-\varepsilon_{\mathrm{j}}\right] /\left[\mu_{\mathrm{j}}^{\mathrm{i}}\left(1-\varepsilon_{\mathrm{j}}\right)\right]=\left[1-\varepsilon_{\mathrm{j}} / \mu_{\mathrm{j}}^{\mathrm{i}}\right] /\left[1-\varepsilon_{\mathrm{j}}\right], \quad$ то $\quad$ неравенство (17) будет иметь вид

$$
1-\varepsilon_{1} / \mu_{1}^{1} \geq \mathrm{D}^{\mathrm{il}}\left(1-\varepsilon_{1} / \mu_{1}^{\mathrm{i}}\right) .
$$

Обозначим через $\mathrm{z}_{1}{ }^{\mathrm{i}}=1-\varepsilon_{1} / \mu_{1}^{\mathrm{i}}, \quad \mathrm{z}_{1}{ }^{1}=1-\varepsilon_{1} / \mu_{1}{ }_{1} ; \quad \mathrm{Z}_{1}^{1}=1-\mathrm{E}_{1} / \mathrm{M}_{1}^{1} ; \mathrm{Z}_{1}^{\mathrm{i}}=1-\mathrm{E}_{1} / \mathrm{M}_{1}^{\mathrm{i}}$; $\hat{\mathrm{Z}}_{1}^{1}=1-\mathrm{E}_{1} / \hat{\mathrm{M}}_{1}^{1} ; \quad \hat{\mathrm{Z}}_{1}^{\mathrm{i}}=1-\mathrm{E}_{1} / \hat{\mathrm{M}}_{1}^{\mathrm{i}}$. Тогда неравенство (10) будет иметь вид $\mathrm{z}_{1}^{1} \geq \mathrm{D}^{\mathrm{i}} \mathrm{z}_{1}^{\mathrm{i}}$. Необходимо показать, что из условия $\mathrm{z}_{1}^{1} \geq \mathrm{D}^{\mathrm{il}} \mathrm{z}_{1}^{\mathrm{i}}$ следует неравенство $\hat{Z}_{1}^{1} \geq \mathrm{D}^{\mathrm{il}} \hat{Z}_{1}^{\mathrm{i}}$. Таким образом, доказательство утверждения сводится к поиску преобразования $\hat{Z}=\phi(z)$ такого, что из выполнения неравенства $\mathrm{z}_{1}^{1} \geq \mathrm{D}^{\mathrm{il}} \mathrm{z}_{1}^{\mathrm{i}}$ следует, что $\phi\left(\mathrm{z}_{1}^{1}\right) \geq \mathrm{D}^{\mathrm{il}} \phi\left(\mathrm{z}_{1}^{\mathrm{i}}\right)$.

Вначале найдем решение следующего функционального уравнения относительно неизвестной функции ф:

$$
\phi\left(z^{1}\right)=D^{i l} \phi\left(z^{i}\right) .
$$

Учитывая, что $\mathrm{z}^{1}=\mathrm{D}^{\mathrm{il}} \mathrm{z}^{\mathrm{i}}$, имеем $\phi\left(\mathrm{D}^{\mathrm{il}} \mathrm{z}\right)=\mathrm{D}^{\mathrm{i}} \phi(\mathrm{z})$. Будем искать решение уравнения (19) в классе непрерывно дифференцируемых функций. Продифференцируем обе части последнего уравнения по $z: D^{\mathrm{il}} \phi^{\prime}\left(\mathrm{D}^{\mathrm{il}} \mathrm{z}\right)=\mathrm{D}^{\mathrm{il}} \phi^{\prime}(\mathrm{z})$. T.к. $\mathrm{D}^{\mathrm{il}} \neq 0$, то $\phi^{\prime}\left(\mathrm{D}^{\mathrm{il}} \mathrm{z}\right)=\phi^{\prime}(\mathrm{z})$. Введем обозначение $\mathrm{g}(\mathrm{z})=\phi^{\prime}(\mathrm{z})$. Тогда для любого $\mathrm{D}^{\mathrm{il}}>0$ имеет место следующее функциональное уравнение: $\mathrm{g}\left(\mathrm{D}^{\mathrm{il}} \mathrm{z}\right)=\mathrm{g}(\mathrm{z})$. При $\mathrm{D}^{\mathrm{i} 1}<1 \mathrm{~g}(\mathrm{x})=\mathrm{g}\left(\mathrm{D}^{\mathrm{i} 1} \mathrm{x}\right)=\mathrm{g}\left[\left(\mathrm{D}^{\mathrm{i} 1}\right)^{2} \mathrm{x}\right]=\ldots=\mathrm{g}\left[\left(\mathrm{D}^{\mathrm{i} 1}\right)^{\mathrm{n}} \mathrm{x}\right]$. Перейдя к пределу при $\mathrm{n} \rightarrow \infty$, получим $\mathrm{g}(\mathrm{z})=\mathrm{g}(0)=$ const. Пусть $\mathrm{g}(\mathrm{z})=\gamma$, т.е. $\phi^{\prime}(\mathrm{z})=\gamma$. Поэтому $\phi(\mathrm{z})=\gamma \mathrm{z}+\beta$, где $\gamma, \beta=$ const. Определим значения констант $\gamma$ и $\beta$. Так как $\mathrm{z}_{1}^{1}=\mathrm{D}^{\mathrm{il}} \mathrm{z}_{1}^{\mathrm{i}}$, то $\gamma \mathrm{z}_{1}^{1}+\beta=\phi\left(z_{1}^{l}\right)=\mathrm{D}^{\mathrm{il}} \phi\left(\mathrm{z}_{1}^{\mathrm{i}}\right)=\mathrm{D}^{\mathrm{il}}\left(\gamma \mathrm{z}_{1}^{1}+\beta\right)=\mathrm{D}^{\mathrm{il}} \gamma \mathrm{z}_{1}^{\mathrm{i}}+\mathrm{D}^{\mathrm{il}} \beta$. При этом $\gamma \mathrm{z}_{1}^{1}+\beta=\gamma \mathrm{D}^{\mathrm{il}} \mathrm{z}_{1}^{\mathrm{i}}+\beta$, тогда $\beta=\mathrm{D}^{\mathrm{i} 1} \beta$ для любого $\mathrm{D}^{\mathrm{i}}$. Следовательно, $\beta=0$ и $\phi(\mathrm{z})=\gamma \mathrm{z}$, a решением уравнения (19) при $\gamma \neq 0$ является функция $\phi(z)=\gamma z$. Поскольку $z_{1}^{1} \geq D^{i l} z_{1}^{i}, D^{i 1}>0$, то для любого $\gamma>0$ выполняется неравенство $\gamma \mathrm{z}_{1}^{1}>\gamma \mathrm{D}^{\mathrm{il}} \mathrm{z}_{1}^{\mathrm{i}}$, которое можно переписать в виде $\phi\left(\mathrm{z}_{1}^{1}\right) \geq \mathrm{D}^{\mathrm{il}} \phi\left(\mathrm{z}_{1}^{\mathrm{i}}\right)$. Отсюда следует, что $\hat{Z}_{1}^{i}=\gamma_{1} z_{1}^{i}$ или $1-\mathrm{E}_{1} / \hat{\mathrm{M}}_{1}^{\mathrm{i}}=\gamma_{1}\left(1-\varepsilon_{1} / \mu_{1}^{\mathrm{i}}\right)$.

Так, скорректированная абсолютная оценка качества $\hat{\mathrm{M}}_{1}^{\mathrm{i}}$ для і-го состояния ВТС вычисляется по следующей формуле:

$$
\hat{\mathrm{M}}_{1}^{\mathrm{i}}=\mathrm{E}_{1} /\left[1-\gamma_{1}\left(1-\varepsilon_{1} / \mu_{1}^{\mathrm{i}}\right)\right] .
$$

Таким же образом осуществляется корректировка остальных частных абсолютных оценок качества. В табл. 3 даны формулы корректировки для некоторых видов этих оценок. 
Таблица 3. Формулы корректировки частых абсолютных квалиметрических оценок

\begin{tabular}{|c|c|c|}
\hline $\begin{array}{c}\text { Номер } \\
\Pi / \Pi\end{array}$ & Способы нормировки & Скорректированная абсолютная оценка \\
\hline 1 & $\mu_{\mathrm{j}}^{\mathrm{i}}=\frac{\mathrm{a}_{\mathrm{j}}}{\mathrm{y}_{\mathrm{j}}^{\mathrm{i}}}, \mathrm{M}_{\mathrm{j}}^{\mathrm{i}}=\frac{\mathrm{A}_{\mathrm{j}}}{\mathrm{y}_{\mathrm{j}}^{\mathrm{i}}}$ & $\begin{array}{c}\hat{\mathrm{M}}_{\mathrm{j}}^{\mathrm{i}}=\mathrm{E}_{\mathrm{j}} /\left[1-\gamma_{\mathrm{j}}\left(1-\varepsilon_{\mathrm{j}} /\left(\mathrm{p}_{\mathrm{j}} \mathrm{M}_{\mathrm{j}}^{\mathrm{i}}\right)\right)\right] \\
\text { где } \mathrm{p}_{\mathrm{j}}=\mathrm{a}_{\mathrm{j}} / \mathrm{A}_{\mathrm{j}}, 0<\gamma_{\mathrm{j}} \leq\left[1-\mathrm{E}_{\mathrm{j}}\right] /\left[1-\varepsilon_{\mathrm{j}} \mathrm{A}_{\mathrm{j}} / \mathrm{a}_{\mathrm{j}}\right]\end{array}$ \\
\hline 2 & $\mu_{j}^{i}=\frac{b_{j}-y_{j}^{i}}{b_{j}-a_{j}}, M_{j}^{i}=\frac{B_{j}-y_{j}^{i}}{B_{j}-A_{j}}$ & $\begin{array}{c}\hat{M}_{j}^{i}=E_{j} /\left[1-\gamma_{j}\left(1-\varepsilon_{j} /\left(p_{j} M_{j}+q_{j}\right)\right)\right] \\
\text { где } p_{j}=\left(B_{j}-A_{j}\right) /\left(b_{j}-a_{j}\right), q_{j}=\left(b_{j}-B_{j}\right) /\left(b_{j}-a_{j}\right), \\
0 \leq \gamma_{j} \leq\left[1-E_{j}\right] /\left[1-\varepsilon_{j}\left(b_{j}-a_{j}\right) /\left(b_{j}-A_{j}\right)\right]\end{array}$ \\
\hline 3 & $\mu_{j}^{i}=\left[\frac{b_{j}-y_{j}^{i}}{b_{j}-a_{j}}\right]^{\beta_{j}}, M_{j}^{i}=\left[\frac{B_{j}-y_{j}^{i}}{B_{j}-A_{j}}\right]^{\beta_{j}}$ & $\begin{array}{c}\hat{M}_{j}^{i}=E_{j} /\left[1-\gamma_{j}\left(1-\varepsilon_{j} /\left[p_{j}\left(M_{j}^{i}\right)^{1 / k}+q_{j}\right]^{k}\right)\right], \\
\text { где } p_{j}=\left(B_{j}-A_{j}\right) /\left(b_{j}-a_{j}\right), q_{j}=\left(b_{j}-B_{j}\right) /\left(b_{j}-a_{j}\right), \\
0<\gamma_{j} \leq\left[1-E_{j}\right] /\left[1-\varepsilon_{j}\left(p_{j}+q_{j}\right)^{k}\right]\end{array}$ \\
\hline
\end{tabular}

Таблица 4. Показатели шума, абсолютные оценки качества окружающей среды, относительные и интегральная оценки акустической дискомфортности первоначального списка объектов шумового мониторинга

\begin{tabular}{|c|c|c|c|c|c|c|c|c|}
\hline $\begin{array}{c}\text { Номер } \\
\text { п/п }\end{array}$ & $\mathrm{y}_{1}$ & $\mathrm{y}_{2}$ & $\mu_{1}$ & $\mu_{2}$ & $\mathrm{~d}_{1}$ & $\mathrm{~d}_{2}$ & $\mathrm{~d}$ & Ранг \\
\hline 1 & 94 & 75 & 0,310 & 0,255 & 0,176 & 0,245 & 0,212 & 8 \\
\hline 2 & 56 & 48 & 0,953 & 0,939 & 0,004 & 0,005 & 0,005 & 1 \\
\hline 3 & 82 & 70 & 0,513 & 0,381 & 0,075 & 0,136 & 0,106 & 5 \\
\hline 4 & 89 & 76 & 0,395 & 0,229 & 0,122 & 0,281 & 0,206 & 7 \\
\hline 5 & 92 & 81 & 0,344 & 0,103 & 0,151 & 0,732 & 0,523 & 9 \\
\hline 6 & 98 & 71 & 0,243 & 0,356 & 0,248 & 0,151 & 0,201 & 6 \\
\hline 7 & 87 & 50 & 0,429 & 0,888 & 0,106 & 0,011 & 0,059 & 2 \\
\hline 8 & 88 & 54 & 0,412 & 0,787 & 0,113 & 0,023 & 0,069 & 4 \\
\hline 9 & 107 & 73 & 0,090 & 0,305 & 0,798 & 0,191 & 0,596 & 10 \\
\hline 10 & 88 & 53 & 0,412 & 0,812 & 0,113 & 0,019 & 0,068 & 3 \\
\hline
\end{tabular}

Покажем применение интегральных оценок $\mathrm{d}^{\mathrm{i}}$ для оценки изменения качества (техногенной безопасности) функционирования ВТС с учетом дополнительной информации (наблюдений) о состоянии «новых» систем на примере мониторинга шумового загрязнения окружающей среды под воздействием военной авиации [8]. Качество функционирования ВТС - техногенную опасность, в данном случае акустическую дискомфортность, приаэродромной территории будем оценивать с точки зрения двух показателей шумового загрязнения окружающей среды: $\mathrm{y}_{1}$ - «эквивалентный уровень звука» и $\mathrm{y}_{2}$ - «максимальный уровень звука».

В табл. 4 представлены исходные данные, промежуточные расчеты и результаты построения интегральной оценки акустической дискомфортности первоначального списка объектов шумового мониторинга. 
Таблица 5. Показатели шума, абсолютные оценки качества окружающей среды, относительные и интегральная оценки акустической дискомфортности объектов шумового мониторинга с дополнительной информацией

\begin{tabular}{|c|c|c|c|c|c|c|c|c|c|c|c|c|}
\hline $\begin{array}{c}\text { Hомер } \\
\text { п/п }\end{array}$ & $\mathrm{y}_{1}$ & $\mathrm{y}_{2}$ & $\mu_{1}$ & $\mathrm{M}_{1}$ & $\hat{\mathrm{M}}_{1}$ & $\mu_{2}$ & $\mathrm{M}_{2}$ & $\hat{\mathrm{M}}_{2}$ & $\hat{\mathrm{D}}_{1}$ & $\hat{\mathrm{D}}_{2}$ & $\hat{\mathrm{D}}$ & Ранг \\
\hline 1 & 94 & 75 & 0,310 & 0,323 & 0,245 & 0,255 & 0,319 & 0,057 & 0,256 & 0,360 & 0,310 & 10 \\
\hline 2 & 56 & 48 & 0,953 & 0,908 & 0,454 & 0,939 & 0,894 & 0,122 & 0,100 & 0,156 & 0,129 & 2 \\
\hline 3 & 82 & 70 & 0,513 & 0,508 & 0,336 & 0,381 & 0,426 & 0,075 & 0,165 & 0,267 & 0,217 & 7 \\
\hline 4 & 89 & 76 & 0,395 & 0,400 & 0,287 & 0,229 & 0,298 & 0,053 & 0,207 & 0,390 & 0,305 & 9 \\
\hline 5 & 92 & 81 & 0,344 & 0,354 & 0,263 & 0,103 & 0,191 & 0,027 & 0,233 & 0,773 & 0,583 & 11 \\
\hline 6 & 98 & 71 & 0,243 & 0,262 & 0,206 & 0,356 & 0,404 & 0,072 & 0,321 & 0,280 & 0,301 & 8 \\
\hline 7 & 87 & 50 & 0,429 & 0,431 & 0,302 & 0,888 & 0,851 & 0,119 & 0,192 & 0,160 & 0,177 & 3 \\
\hline 8 & 88 & 54 & 0,412 & 0,415 & 0,295 & 0,787 & 0,766 & 0,113 & 0,199 & 0,171 & 0,185 & 5 \\
\hline 9 & 107 & 73 & 0,090 & 0,123 & 0,092 & 0,305 & 0,362 & 0,065 & 0,818 & 0,313 & 0,646 & 12 \\
\hline 10 & 88 & 53 & 0,412 & 0,415 & 0,295 & 0,812 & 0,787 & 0,115 & 0,199 & 0,168 & 0,184 & 4 \\
\hline 11 & 109 & 88 & - & 0,092 & 0,092 & - & 0,043 & 0,043 & 0,819 & 0,489 & 0,696 & 13 \\
\hline 12 & 88 & 45 & - & 0,415 & 0,415 & - & 0,957 & 0,957 & 0,117 & 0,001 & 0,061 & 1 \\
\hline 13 & 101 & 79 & - & 0,215 & 0,215 & - & 0,234 & 0,234 & 0,304 & 0,071 & 0,196 & 6 \\
\hline
\end{tabular}

К первоначальному списку объектов шумового мониторинга были добавлены три «новых» наблюдения с номерами 11-13. Результат построения интегральной оценки акустической дискомфортности объектов шумового мониторинга с дополнительной информацией приведен в табл. 5.

Ранжировка первоначального списка объектов шумового мониторинга имеет следующий вид: $2,7,10,8,3,6,4,1,5,9$; а для списка с дополнительной информацией - 12, 2, 7, 10, 8, 13, 3, 6, 4, 1, 5, 9, 11 (полужирным начертанием выделены объекты, добавленные в первоначальный список). Таким образом, для элементов первоначального списка ранжировка сохранилась, т.е. «новые» объекты шумового мониторинга «добавились» в первоначальную ранжировку.

Предлагаемая интегральная квалиметрическая оценка функционирования ВТС является нелинейным (неаддитивным) критерием качества управленческих решений и отличается от аналогов тем, что:

1) имеет содержательную и вероятностную интерпретации, что необходимо для формализации понятия «опасность (риск)» функционирования ВТС;

2) позволяет непосредственно учитывать требования (нормативы) к качеству функционирования ВТС в виде нормативных уровней - нижних предельно допустимых значений по каждому ПК в отдельности;

3) частные показатели качества функционирования ВТС могут быть измерены в различных шкалах (шкале отношений, интервальной шкале, в порядковой шкале, в виде балльных оценок);

4) возможен учет неравноценности частных оценок качества функционирования ВТС на основе определения их весовых коэффициентов;

5) применима в системах мониторинга управления функционированием ВТС.

$$
-247-
$$




\section{Список литературы}

[1] Каплинский А.И., Руссман И.Б., Умывакин В.М. Моделирование и алгоритмизация слабоформализованных задач выбора наилучших вариантов системы. Воронеж: Изд-во Воронеж. гос. ун-та, 1991. 168c. [Kaplinsky A. I., Russman I. B., Umyvakin V. M. Modeling and algorithmization slaboformalizovannykh of problems of the choice of the best options of system. Voronezh publishing house state university, 1991. 168 p. (in Russian)].

[2] Зибров Г.В., Умывакин В.М., Матвиец Д.А. Геоэкологическая квалиметрия природнохозяйственных территориальных систем. Экологические системы и приборы, 2011, 5, 3-9. [Zibrov G. V., Umyvakin V. M., Matviyets D. A. Geoecological kvalimetriya of natural and economic territorial systems. Ecological systems and devices, 2011, 5, 3-9 (in Russian)].

[3] Зибров Г.В., Умывакин В.М., Пахмелкин А.В., Козирацкий А.Ю. Методические аспекты обеспечения экологической безопасности военно-строительных комплексов. Вестник Воен. авиаи. инж. ун-та, 2011, 3(14), 164-169 [Zibrov G. V., Umyvakin V. M., Pakhmelkin A.V., Koziratsky A. Yu. Methodical aspects of ensuring ecological safety of military and construction complexes. Messenger Military aviation engineering university, 2011, 3(14), 164-169 (in Russian)].

[4] Умывакин В.М., Швец А.В., Гедзенко М.О. Квалиметрия экологической опасности территорий военных природно-техногенных систем. Наукоемкие технологии, 2012, 13(3), 34-39 [Umyvakin V. M., Shvets A.V., Gedzenko M. O. Kvalimetriya of ecological danger of territories of military natural and technogenic systems. High technologies, 2012, 13(3), 34-39. (in Russian)].

[5] Harrington E.C.Jr. The desirability function. Industrial quality control, 1965, 21(10), 494498.

[6] Джини К. Средние величиныл. М.: Статистика, 1970. 448 с. [Jeanie К. Average sizes. Moscow, Statistics, 1970. 448 p. (in Russian)].

[7] Яглом А.М., Яглом И.М. Вероятность и информация. М.: Гос. изд-во техн.-теорет. лит. 1957. 160 c. [Yagl A. M., Yagl I. M. Veroyatnost and information. Moscow, State. publishing house tehn.-teort. litas. 1957. 160 p. (in Russian)].

[8] Михайлов В.В., Умывакин В.М., Швец А.В. Методическое обеспечение системы комплексного экологического мониторинга приаэродромных территорий при техногенном воздействии на окружающую среду военной авиации, Академические Жуковские чтения. Системы гидрометеорологического, экологического и специального мониторинга: методические аспекты повышения качества. Воронеж: ВУНЦ ВВС «ВВА», 2014, 147-152. [Mikhaylov V.V., Umyvakin V.M., Shvets A.V. Methodical providing system of complex environmental monitoring of aerodrome environs at technogenic impact on the environment of military aircraft, The Academic Zhukovsky readings. Systems of hydrometeorological, environmental and special monitoring: methodical aspects of improvement of quality. Voronezh: MESC AF «AFA», 2014, P. 147-152 (in Russian)]. 